$\mathbb{T}$ periodica polytechnica

\author{
Social and Management Sciences \\ $19 / 2(2011) 87,96$ \\ doi: 10.3311/pp.so.2011-2.05 \\ web: http://www.pp.bme.hu/so \\ (c) Periodica Polytechnica 2011
}

RESEARCH ARTICLE

\section{An alternative solution to an economic order quantities for recoverable item inventory systems}

\author{
Imre Dobos
}

Received 2011-06-07

\begin{abstract}
In this paper we analyze a reverse logistics inventory model investigated by Teunter (2001). Demand of a product can be satisfied with newly manufactured and remanufactured products. The used products are collected and then returned to the manufacturer of the product. The returned items are inspected and some of the used products are disposed outside. The rate of reuse is decision variable in this model. The goal of the decision maker is to minimize the relevant costs. The aim of this paper is to reexamine the proposed model and to improve some small mistakes. First, we show that it is comfortable to determine the batch numbers of the manufacturing and remanufacturing activities, because these decision variables are integer, rather than the manufacturing and remanufacturing lot sizes. Secondly, we show that there exist optimal solutions for the batch numbers inside of the possible set, i.e. both manufacturing and remanufacturing batches could be higher than one. Thirdly, the above mentioned paper have not analyzed a case with one batch numbers both manufacturing and remanufacturing. This case can occur for some parameters.
\end{abstract}

\section{Keywords}

Reverse Logistics · Closed-loop supply chain · Lotsizing . EOQ $\cdot$ Optimization

\section{Acknowledgement}

The author gratefully acknowledges the financial supports from the TÁMOP-4.2.1.B-09/KMR-2010-0005 research program and the Deutscher Akademischer Austauschdienst (DAAD).

\section{Introduction}

Quantitative models for inventory systems with product recovery management provide an actual generalization of classical EOQ models. The classical EOQ model analyzes one product inventory systems. The difficulty of recovery system is that manufactured (purchased) items must be handled simultaneously in a manufacturing-remanufacturing cycle. A number of authors have proposed such models, like Schrady [4], Nahmias and Rivera [2], and Richter [3]. Our paper deals with one of these proposals, we investigate the model of Teunter [5].

The goal of the paper is to reconsider the Teunter's model. First, the explicit model will be discussed and a way of solution is offered for this model, because the author has neglected to describe the explicit model for a general inventory holding policy, i.e. with explicit manufaturing and remanufacturing batch numbers. The model is solved in two ways. In the first method we eliminate the batch numbers, in order to calculate the manufacturing and remanufacturing lot sizes. This way of solution is common in the EOQ-type inventory literature, but it makes difficult to determine the integer batch numbers for manufacturing and remanufacturing. The second method offers to eliminate the lot sizes, and then to determine the batch numbers. This way leads to a meta-model offered by Richter [3], and analyzed mathematically by Dobos and Richter [1]. This model makes it easier to calculate the optimal reuse rate of the model.

The paper organizes, as follows. In the second section the model is shown and the cost function of the problem is constructed. Section three presents the possible ways to solve the problem, and shows the integer solution of the model. The next part deals with the determination of the optimal reuse rate, and last we sumatize the results of the paper.

\section{The model and the cost function}

Teunter [5] has investigated in his model the following activities:

- remanufacturing,

\section{Imre Dobos}

Institute of Business Economics, Corvinus University Budapest, H-1093 Budapest, Fővám tér 8., Hungary

e-mail:imre.dobos@uni-corvinus.hu
- disposal and

- manufacturing. 
Let a cycle be deined, as the above-mentioned schedule of activities with fixed batch sizes for manufacturing and remanufacturing. In a planning period there is only one cycle. (This can be proved very easily by grouping the remanufacturing and manufacturing lots.)

The goal of the decision maker is to minimize the relevant costs for manufacturing and remanufacturing. There are EOQoriented setup and holding costs for remanufacturing and manufacturing, and linear production and remanufacturing costs, linear disposal cost and holding cost for non-serviceable items.

The notations of the model are the following:

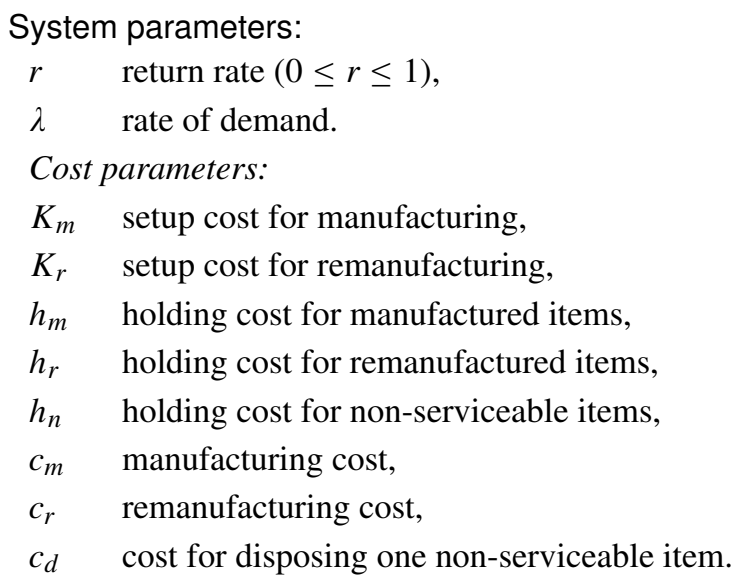

\section{Decision variables:}

$Q_{m}$ batch size for manufacturing,

$Q_{r} \quad$ batch size for remanufacturing,

$M \quad$ number of manufacturing batches, positive integer,

$R \quad$ number of remanufacturing batches, positive integer,

$T \quad$ length of the product recovery cycle,

$u \quad$ reuse rate $(0 \leq u \leq r)$.

We assume that all parameters and the decisions variables are nonnegative numbers. We will describe the mathematical model with some application.

First we examine stock-flow balance of serviceable and recoverable stocks. Eq. (1) shows that the sum of manufactured and remanufacted products must cover the demand in a cycle. Eq. (2) is the relation between the returned products and the use of these products for remanufacturing and disposal. The material flow of the model is shown in Fig. 1

$$
\begin{gathered}
M Q_{m}+R Q_{r}=\lambda T \\
R Q_{r}+(r-u) \lambda T=r T
\end{gathered}
$$

From the linear systems (1) and (2) we can write two separate equations for the manufacturing and remanufacturing batches:

$$
M Q_{m}=(1-u) \lambda T
$$

and

$$
R Q_{r}=u \lambda T
$$

If the reuse rate is equal to zero, i.e. $u=0$, then the remanufacturing lot size is zero, i.e. $Q_{r}=0$ in relation (4). It means that all returned parts are disposed, there is no reuse in system and the management problem turns into a simple inventory problem. Another interesting case is, if the return rate is equal to reuse rate $(u=r)$. This case shows an example, when all returned parts are reused and there is no disposal activity. Identity (3) and (4) will be useful to create our cost function.

Now we construct the total cost function. We do it in two steps. In the first step we investigate the inventory holding cost function $H\left(Q_{m}, Q_{r}, T, M, R, u\right)$ for serviceable and nonserviceable parts. In the second step we describe the linear costs $L\left(Q_{m}, Q_{r}, T, M, R, u\right)$ of manufacturing, remanufacturing, and disposal.

Let us now calculate the inventory holding costs $H\left(Q_{m}, Q_{r}\right.$, $T, M, R, u)$. The inventory holding policy is shown in Fig. 2. This inventory holding policy presents a manufacturing/remanufacturing strategy, i.e. a cycle. A manufacturing/remanufacturing cycle is an order of these activities. In Fig. 2 we have shown a cycle beginning with given remanufacturing batches and then followed with given manufacturing batches. As in the classical EOQ model, these fixed cycles are repeated infinitely. Of course, we can change the order of the activites, i.e. a cycle can begin with manufacturing and followed with remanufacturing, but this order does not change the inventory holding costs. This policy is a predetermined policy and we look for the optimal parameters $\left(Q_{m}, Q_{r}, T, M, R, u\right)$ of this strategy. Let us assume that the inventory level functions for a known strategy are function $I^{s}(t)$ for serviceable stock and function $I^{r}(t)$ for recoverable stock, $0 \leq t \leq T$. The inventory holding costs are the area below this functions, i.e.

$$
\begin{gathered}
H\left(Q_{m}, Q_{r}, M, R, u\right)= \\
h_{r} \int_{0}^{T u} I^{s}(t) d t+h_{m} \int_{T u}^{T} I^{s}(t) d t+h_{n} \int_{0}^{T} I^{r}(t) d t .
\end{gathered}
$$

Now we use the property of the inventory policy that the sum of serviceable and recoverable products is a monotone decreasing, linear and continuous function of time in the remanufacturing cycle. So the inventory cycle can be divided into two subcycles.

1 the demand is satisfied from remanufacturing, and the recoverable stock is positive. The length of this interval is equal to $T u-\frac{Q_{r}}{\lambda}$

2 The demand is satisfied from the last remanufacturing batch and from manufacturing, and the stock level of recoverable items is monotone nondecreasing. A remanufacturing batch is used in an interval length of $\frac{Q_{r}}{\lambda}$. The length of this subcycle is $T(1-u)+\frac{Q_{r}}{\lambda}$.

So the inventory holding cost function can be expressed with 


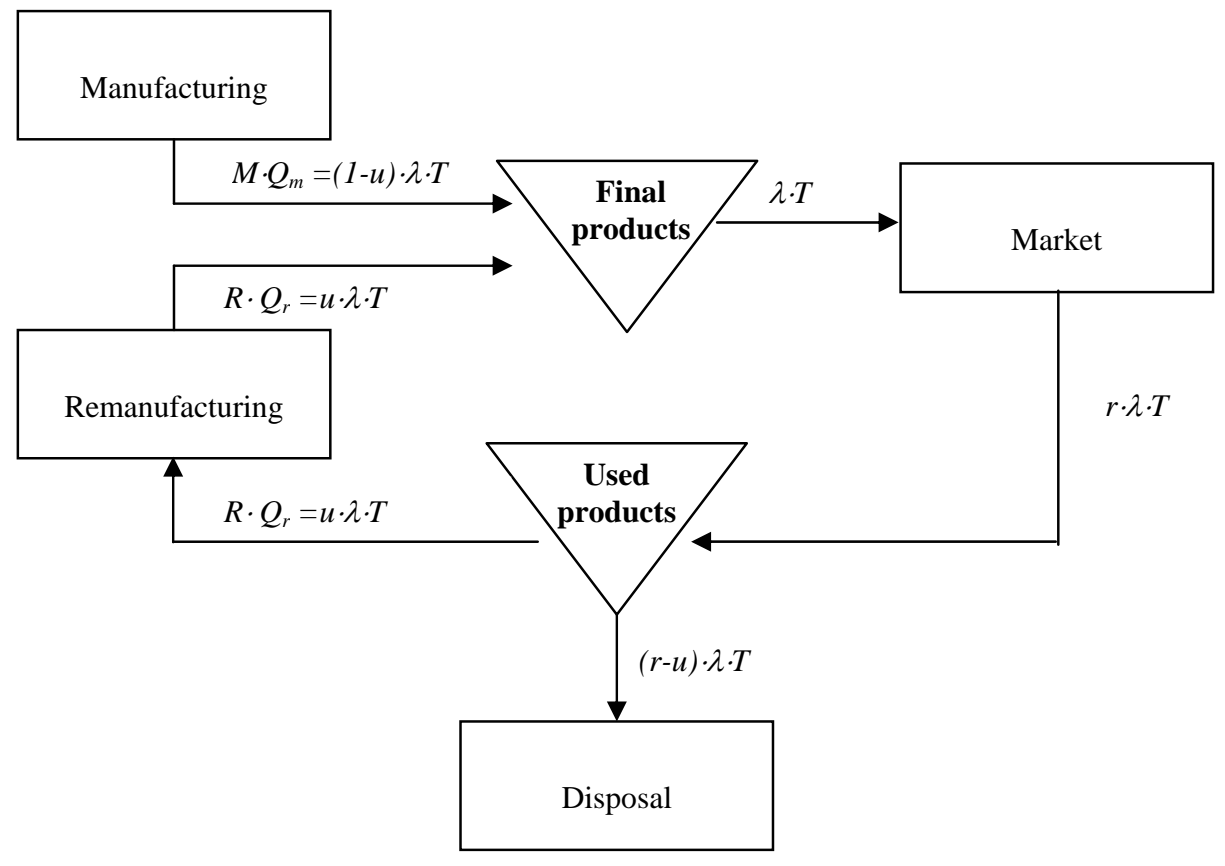

Fig. 1. Material flow in the model

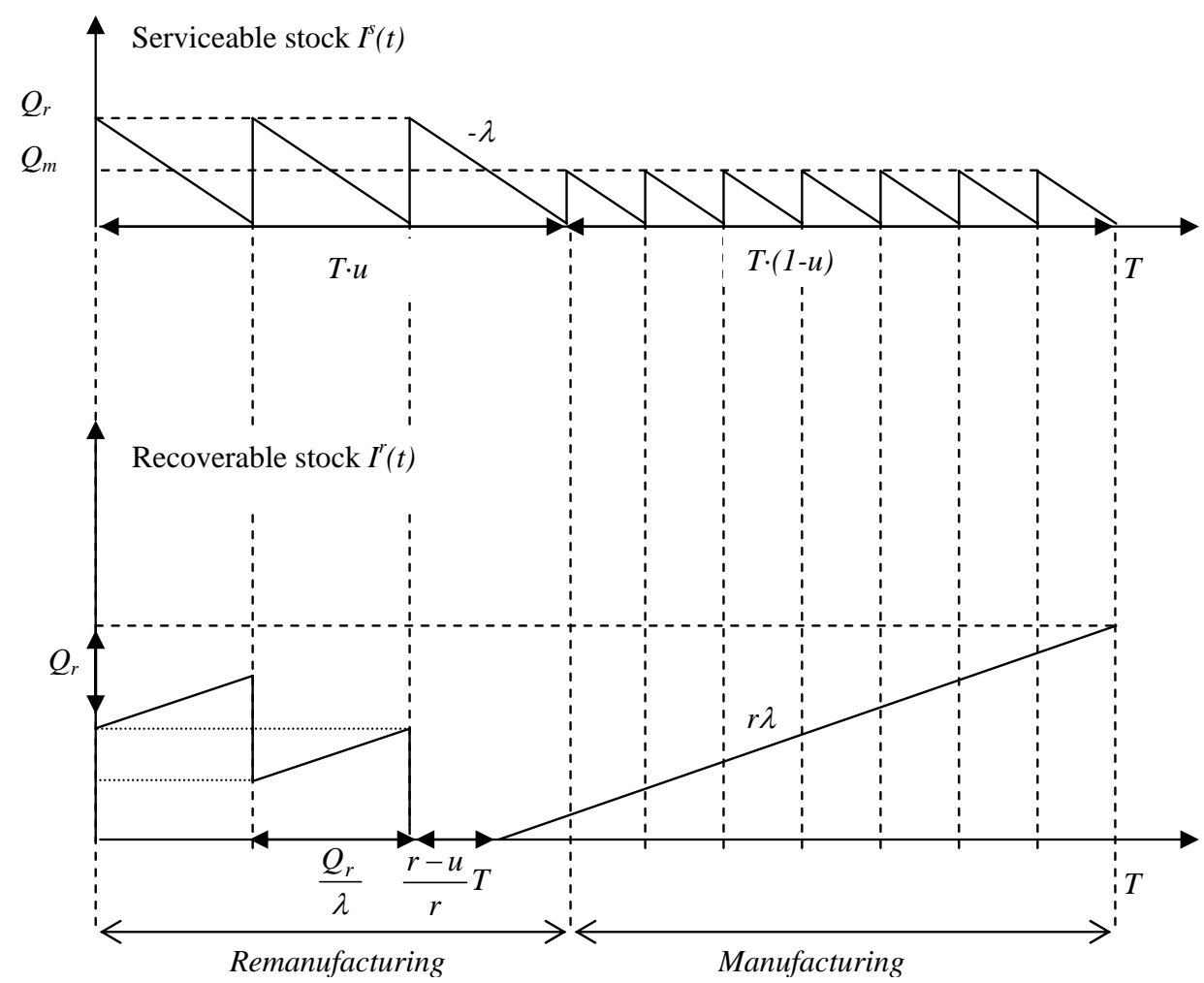

Fig. 2. Modeling the inventory policy $(R=3, M=7)$

the help of the cycles, as

$$
\begin{aligned}
& H\left(Q_{m}, Q_{r}, M, R, u\right)= \\
& \left(h_{r}-h_{n}\right) \int_{0}^{T u-\frac{Q_{r}}{\lambda}} I^{s}(t) d t+h_{r} \\
& \int_{T u-\frac{Q_{r}}{\lambda}}^{T u} I^{S}(t) d t+h_{m} \int_{T u}^{T} I^{S}(t) d t+ \\
& h_{n}^{T u-\frac{Q_{r}}{\lambda}}\left[I^{S}(t)+I^{r}(t)\right] d t+h_{n} \int_{T u-\frac{Q_{r}}{\lambda}}^{T} I^{r}(t) d t
\end{aligned}
$$

We must now calculate the five integrals. The first integral consists of $R-1$ pieces of remanufacturing batches. The costs are $\left(h_{r}-h_{n}\right) \int_{0}^{T u-\frac{Q_{r}}{\lambda}} I^{s}(t) d t=(R-1)\left(h_{r}-h_{n}\right) \frac{Q_{r}^{2}}{2 \lambda}$. The second integral is only a remanufacturing batch $h_{r} \int_{T u-\frac{Q_{r}}{\lambda}}^{T u} I^{s}(t) d t=$ $h_{r} \frac{Q_{r}^{2}}{2 \lambda}$. The third value is the cost of inventory holding of manu- 
factured products, which consists of $M$ batches $h_{m} \int_{T u}^{T} I^{S}(t) d t=$ $M h_{m} \frac{Q_{m}^{2}}{2 \lambda}$. The computation of the fourth integral is a little bit complicated. We have pointed out that the sum of the inventory levels $I^{s}(t)+I^{r}(t)$ is a monotone decreasing linear function. The tangent of this linear function is $(1-r) \lambda$. In point of time $T u-\frac{Q_{r}}{\lambda}$ this function has a value of $Q_{r}$. With this assumption the value of the integral is $h_{n} \int_{0}^{T u-\frac{Q_{r}}{\lambda}}\left[I^{s}(t)+I^{r}(t)\right] d t=$ $h_{n}\left[Q_{r}+(1-r) \frac{\lambda}{2}\left(T u-\frac{Q_{r}}{\lambda}\right)\right]\left(T u-\frac{Q_{r}}{\lambda}\right)$. The fifth, last integral is $h_{n} \int_{T u-\frac{Q_{r}}{2}}^{T} I^{r}(t) d t=h_{n} r \frac{\lambda}{2}\left(\frac{1-r}{r} T u+\frac{Q_{r}}{\lambda}\right)^{2}$.

Summing up the integrals and with elementary calculations, we have the following expression for the inventory holding costs:

$$
\begin{gathered}
H\left(Q_{m}, Q_{r}, T, M, R, u\right)= \\
R h_{r} \frac{Q_{r}^{2}}{2 \lambda}+M h_{m} \frac{Q_{m}^{2}}{2 \lambda}+h_{n} \frac{Q_{r}^{2}}{2 \lambda}\left\{R^{2} \frac{1-r}{r}+R\right\} .
\end{gathered}
$$

In this expression we have applied from Eq. (4) that $T u=\frac{R Q_{r}}{\lambda}$.

The linear costs of manufacturing, remanufacturing, and disposal can be calculated very easily:

$$
\begin{aligned}
& L\left(Q_{m}, Q_{r}, T, M, R, u\right)=c_{m} M Q_{m}+c_{r} R Q_{r}+c_{d} \lambda T(r-u)= \\
& T \lambda\left[u\left(c_{r}-c_{m}-c_{d}\right)+\left(c_{m}+c_{d} r\right)\right] \\
& \text { Now we can formulate the average cost function }
\end{aligned}
$$
$C_{a}\left(Q_{m}, Q_{r}, T, M, R, u\right)$ summing up the total inventory holding costs (setup and inventory holding costs) and the linear manufacturing, remanufacturing, and disposal costs, and divided with the length of the cycle:

$$
\begin{aligned}
& C_{a}\left(Q_{m}, Q_{r}, T, M, R, u\right)= \\
& =\frac{R K_{r}+R h_{r} \frac{Q_{r}^{2}}{2 \lambda}+M K_{m}+M h_{m} \frac{Q_{m}^{2}}{2 \lambda}+h_{n} \frac{Q_{r}^{2}}{2 \lambda}\left\{R^{2} \frac{1-r}{r}+R\right\}}{T}+ \\
& +u \lambda\left(c_{r}-c_{m}-c_{d}\right)+\lambda\left(c_{m}+c_{d} r\right)
\end{aligned}
$$

This way we have constructed a non-linear mixed-integer mathematical programming problem:

$$
C_{a}\left(Q_{m}, Q_{r} T, M, R, u\right) \rightarrow \min
$$

such that

$$
\begin{aligned}
& M Q_{m}=(1-u) \lambda T, \\
& R Q_{r}=u \lambda T, \\
& 0 \leq u \leq r,
\end{aligned}
$$

$Q_{m} \geq 0, Q_{r} \geq 0, T>0, M, R$ positive integers

The problem $(\mathrm{P})$ consists of two subproblems. The first problem is to determine the optimal inventory related decision variables $\left(Q_{m}, Q_{r}, T, M, R\right)$, and the second problem is to calculate the optimal reuse rate $u$. Of course, we can change the order of the manipulation beginning with the optimal reuse rate. For the sake of simplicity we have decided to determine first the inventory related variables. In the next section we will solve the problem for the relevant variables.

\section{Determination of the optimal inventory related deci- sion variables}

In this section we solve problem $(\mathrm{P})$ in two different way. The difference is the order of eliminating the continuous variables from the cost function using Eqs. (3) and (4). The first method offers to eliminate the integer variables $R$ and $M$, in order to express the manufacturing and remanufacturing lot sizes. Second method suggests elimination of the lot sizes, in order to investigate an integer programming problem. The reuse rate $u$ is parameter in these problems. Let us follow this two ways.

\subsection{Elimination of batch numbers $\boldsymbol{R}$ and $\boldsymbol{M}$}

In this case the author will express the cost function in dependence on the lot sizes $Q_{m}$ and $Q_{r}$, so he must substitute the number of batches in the goal function, i.e. $M=\frac{(1-u) \lambda T}{Q_{m}}$, and $R=\frac{u \lambda T}{Q_{r}}$, which are not smaller than one. With the help of this substitution we can reformulate the problem $(\mathrm{P})$ into the problem $\left(\mathrm{P}^{T}\right)$ as

$$
\begin{aligned}
& u\left(\frac{\lambda K_{r}}{Q_{r}}+\frac{h_{r}+h_{n}}{2} Q_{r}\right)+ \\
& (1-u)\left(\frac{\lambda K_{m}}{Q_{m}}+\frac{h_{m}}{2} Q_{m}\right)+ \\
& \frac{h_{n}}{2} u^{2} \lambda T\left(\frac{1}{r}-1\right)+ \\
& +u \lambda\left(c_{r}-c_{m}-c_{d}\right)+\lambda\left(c_{m}+c_{d} r\right) \rightarrow \min \\
& \text { such that } \\
& \frac{(1-u) \lambda T}{Q_{m}} \geq 1, \\
& \frac{u \lambda T}{Q_{r}} \geq 1, \\
& Q_{m} \geq 0, Q_{r} \geq 0, T>0 .
\end{aligned}
$$

This new cost function is linear in the length of the cycle, and for this length there is a lower bound. It means that, $T=\frac{Q_{r}}{u \lambda}$ and/or $T=\frac{Q_{m}}{(1-u) \lambda}$. If the first inequality is equation, i.e. $R^{o}=1$, then the cost function is

$$
\begin{aligned}
& u\left(\frac{\lambda K_{r}}{Q_{r}}+\frac{h_{r}+\frac{1}{r} h_{n}}{2} Q_{r}\right)+(1-u) \\
& \left(\frac{\lambda K_{m}}{Q_{m}}+\frac{h_{m}}{2} Q_{m}\right)+ \\
& +u \lambda\left(c_{r}-c_{m}-c_{d}\right)+\lambda\left(c_{m}+c_{d} r\right)
\end{aligned}
$$

The optimal lot sizes are in this case $Q_{r}^{o}=\sqrt{\frac{2 \lambda K_{r}}{h_{r}+\frac{1}{r} h_{n}}}$, and $Q_{m}^{o}=\sqrt{\frac{2 \lambda K_{m}}{h_{m}}}$. From these equalities it follows that $\frac{(1-u) \lambda T}{Q_{m}^{o}} \geq$ $\frac{u \lambda T}{Q_{r}^{o}}=1$, i.e. $(1-u) Q_{r}^{o} \geq u Q_{m}^{o}$. This asssumption is held after substitution the optimal lot sizes in the inequality, if

$$
0 \leq u \leq \min \left\{\frac{\sqrt{K_{r} h_{m}}}{\sqrt{K_{r} h_{m}}+\sqrt{K_{m}\left(h_{r}+\frac{1}{r} h_{n}\right)}} ; r\right\} .
$$

The cost function is now after substitution the lot sizes in the cost function

$$
u \sqrt{2 \lambda K_{r}\left(h_{r}+\frac{1}{r} h_{n}\right)}+(1-u) \sqrt{2 \lambda K_{m} h_{m}}+
$$


$+u \lambda\left(c_{r}-c_{m}-c_{d}\right)+\lambda\left(c_{m}+c_{d} r\right)$. The optimal cycle time is now $T^{o}=\frac{1}{u} \sqrt{\frac{2}{\lambda} \frac{K_{r}}{h_{r}+\frac{1}{r} h_{n}}}$.

If the second inequality is an equation, i.e. $M^{o}=1$, then the cost function is

$$
\begin{aligned}
& u\left(\frac{\lambda K_{r}}{Q_{r}}+\frac{h_{r}+h_{n}}{2} Q_{r}\right)+(1-u) . \\
& \left(\frac{\lambda K_{m}}{Q_{m}}+\frac{h_{m}+\frac{u^{2}}{(1-u)^{2}}\left(\frac{1}{r}-1\right) h_{n}}{2} Q_{m}\right)+ \\
& +u \lambda\left(c_{r}-c_{m}-c_{d}\right)+\lambda\left(c_{m}+c_{d} r\right)
\end{aligned}
$$

The optimal lot sizes for this second model are $Q_{r}^{o}=\sqrt{\frac{2 \lambda K_{r}}{h_{r}+h_{n}}}$, and $Q_{m}^{o}=(1-u) \sqrt{\frac{2 \lambda K_{m}}{(1-u)^{2} h_{m}+u^{2}\left(\frac{1}{r}-1\right) h_{n}}}$. From the first two inequalities of the problem $\left(\mathrm{P}^{T}\right)$ it follows that in this case $\frac{u \lambda T}{Q_{r}^{o}} \geq \frac{(1-u) \lambda T}{Q_{m}^{o}}=1$, i.e. $u Q_{m}^{o} \geq(1-u) Q_{r}^{o}$. This last assumption can be reformulated with the help of optimal lot sizes after substitution and some calculations as $\frac{\sqrt{K_{r} h_{m}}}{\sqrt{K_{r} h_{m}}+\sqrt{K_{m}\left(h_{r}+h_{n}\right)-K_{r} h_{n}\left(\frac{1}{r}-1\right)}} \leq u \leq r$. This interval for $u$ exists, if the inequality $\frac{\sqrt{K_{r} h_{m}}}{\sqrt{K_{r} h_{m}}+\sqrt{K_{m}\left(h_{r}+h_{n}\right)-K_{r} h_{n}\left(\frac{1}{r}-1\right)}} \leq r$ holds. We can calculate this smallest value of $u$.

The cost function has the next form after the substitution the optimal lot sizes:

$$
\begin{aligned}
& u \sqrt{2 \lambda K_{r}\left(h_{r}+h_{n}\right)}+ \\
& \sqrt{2 \lambda K_{m}\left[(1-u)^{2} h_{m}+u^{2}\left(\frac{1}{r}-1\right) h_{n}\right]}+. \\
& +u \lambda\left(c_{r}-c_{m}-c_{d}\right)+\lambda\left(c_{m}+c_{d} r\right)
\end{aligned}
$$

This model depends only on the reuse rate $u$. The optimal length of a cycle is

$$
T^{o}=\sqrt{\frac{2}{\lambda} \frac{K_{m}}{(1-u)^{2} h_{m}+u^{2}\left(\frac{1}{r}-1\right) h_{n}}} .
$$

These two cost functions and lot sizes for manufacturing and remanufacturing are the same as those of Teunter [4]. We have formulated necessary conditions, when to use these models in dependence on the reuse rate $u$. We see that there is no lot sizes for manufacturing and remanufacturing, if

$$
\begin{gathered}
\min \left\{\frac{\sqrt{K_{r} h_{m}}}{\sqrt{K_{r} h_{m}}+\sqrt{K_{m}\left(h_{r}+h_{n} \frac{1}{r}\right)}}, r\right\} \leq u \leq \\
\frac{\sqrt{K_{r} h_{m}}}{\sqrt{K_{r} h_{m}}+\sqrt{K_{m}\left(h_{r}+h_{n}\right)-K_{r} h_{n}\left(\frac{1}{r}-1\right)}} .
\end{gathered}
$$

The question is how to solve the model on this interval of the reuse rate. To answer these questions we must generalize the offered model with the case, when both numbers of batches for manufacturing and remanufacturing are equal to one. We will see that this case is investigated in the model suggested by Richter [3], as well.
The not investigated case is $M^{o}=R^{o}=1$. For this case $T=\frac{Q_{m}}{(1-u) \lambda}=\frac{Q_{r}}{u \lambda}$, and $\frac{u \lambda T}{Q_{r}}=\frac{(1-u) \lambda T}{Q_{m}}=1$. Let us know substitute expressions $T=\frac{Q_{m}}{(1-u) \lambda}$, and $Q_{r}=\frac{u}{1-u} Q_{m}$ in the cost function. Then we have

$$
\begin{aligned}
& (1-u) \frac{\lambda\left(K_{r}+K_{m}\right)}{Q_{m}}+\frac{1}{1-u} \frac{1}{2} . \\
& {\left[\left(h_{r}+\frac{1}{r} h_{n}\right)^{2} u^{2}+h_{m}(1-u)^{2}\right] Q_{m}+} \\
& +u \lambda\left(c_{r}-c_{m}-c_{d}\right)+\lambda\left(c_{m}+c_{d} r\right)
\end{aligned}
$$

The optimal lot sizes are for this third case $Q_{m}^{o}=(1-u) \sqrt{\frac{2 \lambda\left(K_{r}+K_{m}\right)}{\left[\left(h_{r}+\frac{1}{r} h_{n}\right) u^{2}+h_{m}(1-u)^{2}\right]}}$, and $Q_{r}^{o}=$ $u \sqrt{\frac{2 \lambda\left(K_{r}+K_{m}\right)}{\left[\left(h_{r}+\frac{1}{r} h_{n}\right) u^{2}+h_{m}(1-u)^{2}\right]}}$. This type of model is valid, if

$$
\begin{gathered}
\frac{\sqrt{K_{r} h_{m}}}{\sqrt{K_{r} h_{m}}+\sqrt{K_{m}\left(h_{r}+h_{n} \frac{1}{r}\right)}} \leq u \leq \\
\frac{\sqrt{K_{r} h_{m}}}{\sqrt{K_{r} h_{m}}+\sqrt{K_{m}\left(h_{r}+h_{n}\right)-K_{r} h_{n}\left(\frac{1}{r}-1\right)}} .
\end{gathered}
$$

The modified cost function is

$$
\begin{gathered}
\sqrt{2 \lambda\left(K_{r}+K_{m}\right)\left[\left(h_{r}+\frac{1}{r} h_{n}\right) u^{2}+h_{m}(1-u)^{2}\right]}+ \\
u \lambda\left(c_{r}-c_{m}-c_{d}\right)+\lambda\left(c_{m}+c_{d} r\right),
\end{gathered}
$$

and the optimal length of a cycle is

$$
T^{o}=\sqrt{\frac{2}{\lambda} \frac{K_{r}+K_{m}}{\left[\left(h_{r}+\frac{1}{r} h_{n}\right) u^{2}+h_{m}(1-u)^{2}\right]}} .
$$

The optimal continuous solution of this method for lot sizes, number of batches and cycle time is in dependence on the reuse rate $u$.

Theorem 1 The optimal continuous solution for the inventory related costs and cost function are

I If $0 \leq u \leq \min \left\{\frac{\sqrt{K_{r} h_{m}}}{\sqrt{K_{r} h_{m}}+\sqrt{K_{m}\left(h_{r}+\frac{1}{r} h_{n}\right)}} ; r\right\}$, then

$$
\begin{gathered}
Q_{r}^{o}=\sqrt{\frac{2 \lambda K_{r}}{h_{r}+\frac{1}{r} h_{n}}}, \\
Q_{m}^{o}=\sqrt{\frac{2 \lambda K_{m}}{h_{m}}}, \quad T^{o}=\frac{1}{u} \sqrt{\frac{2}{\lambda} \frac{K_{r}}{h_{r}+\frac{1}{r} h_{n}}}, \\
R(u)=1, M(u)=\frac{1-u}{u} \sqrt{\frac{K_{r} h_{m}}{K_{m}\left(h_{r}+h_{n}\right)+K_{m} h_{n} \frac{1-r}{r}}}, \\
C^{T}(u)=u \sqrt{2 \lambda K_{r}\left(h_{r}+\frac{1}{r} h_{n}\right)+} \\
(1-u) \sqrt{2 \lambda K_{m} h_{m}}+u \lambda\left(c_{r}-c_{m}-c_{d}\right)+\lambda\left(c_{m}+c_{d} r\right) .
\end{gathered}
$$




$$
\begin{aligned}
& 2 \text { If } \frac{\sqrt{K_{r} h_{m}}}{\sqrt{K_{r} h_{m}}+\sqrt{K_{m}\left(h_{r}+h_{n} \cdot \frac{1}{r}\right)}} \leq u \leq \\
& \frac{\sqrt{K_{r} h_{m}}}{\sqrt{K_{r} h_{m}}+\sqrt{K_{m}\left(h_{r}+h_{n}\right)-K_{r} h_{n}\left(\frac{1}{r}-1\right)}} \text {, then, } \\
& Q_{m}^{o}=(1-u) \sqrt{\frac{2 \lambda\left(K_{r}+K_{m}\right)}{\left[\left(h_{r}+\frac{1}{r} h_{n}\right) u^{2}+h_{m}(1-u)^{2}\right]}}, \\
& Q_{r}^{o}=u \sqrt{\frac{2 \lambda\left(K_{r}+K_{m}\right)}{\left[\left(h_{r}+\frac{1}{r} h_{n}\right) u^{2}+h_{m}(1-u)^{2}\right]}}, \\
& T^{o}=\sqrt{\frac{2}{\lambda} \frac{K_{r}+K_{m}}{\left[\left(h_{r}+\frac{1}{r} h_{n}\right) u^{2}+h_{m}(1-u)^{2}\right]}}, R(u)=1, M(u)=1 \text {, } \\
& C^{T}(u)= \\
& \sqrt{2 \lambda\left(K_{r}+K_{m}\right)\left[\left(h_{r}+\frac{1}{r} h_{n}\right) u^{2}+h_{m}(1-u)^{2}\right]} \\
& +u \lambda\left(c_{r}-c_{m}-c_{d}\right)+\lambda\left(c_{m}+c_{d} r\right) . \\
& 3 \text { If } \frac{\sqrt{K_{r} h_{m}}}{\sqrt{K_{r} h_{m}}+\sqrt{K_{m}\left(h_{r}+h_{n}\right)-K_{r} h_{n}\left(\frac{1}{r}-1\right)}} \leq u \leq r \text {, then } \\
& Q_{r}^{o}=\sqrt{\frac{2 \lambda K_{r}}{h_{r}+h_{n}}} \\
& Q_{m}^{o}=(1-u) \sqrt{\frac{2 \lambda K_{m}}{(1-u)^{2} h_{m}+u^{2}\left(\frac{1}{r}-1\right) h_{n}}} \\
& R(u)=u \sqrt{\frac{K_{m}\left(h_{r}+h_{n}\right)}{K_{r} h_{m}(1-u)^{2}+K_{r} h_{n} \frac{1-r}{r} u^{2}}} \quad M(u)=1, \\
& C^{T}(u)=u \sqrt{2 \lambda K_{r}\left(h_{r}+h_{n}\right)}+ \\
& \sqrt{2 \lambda K_{m}\left[(1-u)^{2} h_{m}+u^{2}\left(\frac{1}{r}-1\right) h_{n}\right]}+ \\
& u \lambda\left(c_{r}-c_{m}-c_{d}\right)+\lambda\left(c_{m}+c_{d} r\right) .
\end{aligned}
$$

Remark. In the theorem 1 we can see, if the reuse rate is relatively small, then only one remanufacturing batch occurs in a recovery cycle. Nevertheless, if the reuse rate is relatively high, then only one manufacturing batch is used in the cycle and the rest of the demand is satisfied from remanufactured products. This result can be relevant for practical application. If the collection rate of used products is low, then it is enough to plan inly one remanufacturing batch in a planning period, and inversely. If the collection rate and the reuse rate is high, than the demand for the new items can be satisfied from remanufactured products and the lacked amount of items to manufacture.

Let us now analyze the optimal solution in dependence of the reuse rate $u$ and return rate $r$. The possible sets of parameters of the three cases are examined parametrically. Let us define the following two functions:

$$
u_{1}(r)=\frac{\sqrt{K_{r} h_{m}}}{\sqrt{K_{r} h_{m}}+\sqrt{K_{m}\left(h_{r}+h_{n} \frac{1}{r}\right)}},
$$

and

$$
u_{2}(r)=\frac{\sqrt{K_{r} h_{m}}}{\sqrt{K_{r} h_{m}}+\sqrt{K_{m}\left(h_{r}+h_{n}\right)-K_{r} h_{n}\left(\frac{1}{r}-1\right)}} .
$$

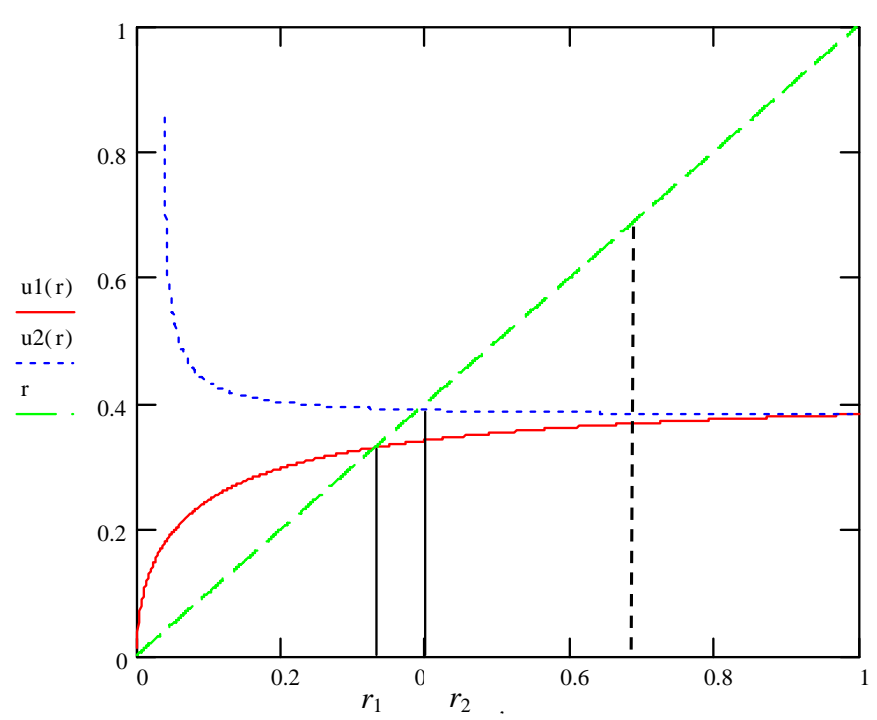

Fig. 3. The possible set of parameters $\boldsymbol{u}$ and $\boldsymbol{r}$

It is easy to see that function $u_{1}(r)$ is smaller than function $u_{2}(r)$, but $u_{1}(1)=u_{2}(1)$.

If we draw these two functions, then we get Fig. 3 .

Fig. 3 shows points $r_{1}$ and $r_{2}$. These two points are the switching points between the solution regions. If the known return rate $r$ is smaller than value $r_{1}$, then only case (1) of theorem 1 occurs in the solution of the problem $\left(\mathrm{P}^{T}\right)$. If reuse rate $r$ is between $r_{1}$ and $r_{2}$, then cases 1 and 2 occur only. If reuse rate $u$ is greater than $r_{2}$, then all three cases are in the optimal solution. The cost function $C^{T}(u)$ is continuous at points $r_{1}$ and $r_{2}$. The dotted vertical line of Fig. 3 shows a general case, when all three cases occur in the solution for $u$.

The continuous solution of problem $\left(\mathrm{P}^{T}\right)$ is known with these last expressions. The question is now, how to determine the integer batch numbers for manufacturing and remanufacturing. This solution method does not give algorithm to determine the integer solution for problem $\left(\mathrm{P}^{T}\right)$.

\subsection{Elimination of lot sizes $Q_{r}$ and $Q_{m}$}

We can follow an other way to solve the problem. Let us substitute the manufacturing and remanufacturing lot sizes $Q_{m}=\frac{(1-u) \lambda T}{M}$ and $Q_{r}=\frac{u \lambda T}{R}$ in the cost function from Eqs. (3) and (4). After substitution the problem has the next form:

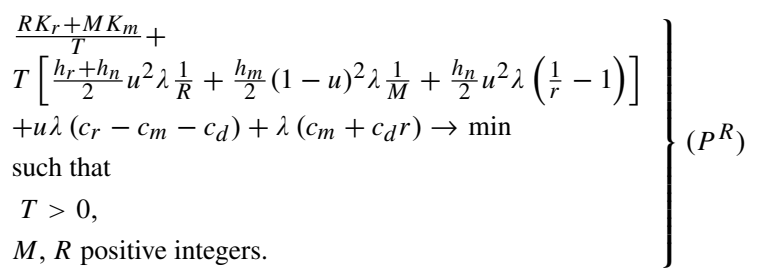

The cost function is now convex in the length of the cycle, so the optimal length can be calculated as follows:

$$
T^{o}(M, R, u)=
$$




$$
\sqrt{\frac{R K_{r}+M K_{m}}{\frac{h_{r}+h_{n}}{2} u^{2} \lambda \frac{1}{R}+\frac{h_{m}}{2}(1-u)^{2} \lambda \frac{1}{M}+\frac{h_{n}}{2} u^{2} \lambda\left(\frac{1}{r}-1\right)}} .
$$

After substitution the optimal length in the cost function, we have the following cost function $C_{I}(R, M, u)$ :

$$
\begin{aligned}
& C_{I}(M, R, u)= \\
& \sqrt{\left(R K_{r}+M K_{m}\right)\left[\frac{h_{r}+h_{n}}{2} u^{2} \lambda \frac{1}{R}+\frac{h_{m}}{2}(1-u)^{2} \lambda \frac{1}{M}+\frac{h_{n}}{2} u^{2} \lambda\left(\frac{1}{r}-1\right)\right]}+ \\
& +u \lambda\left(c_{r}-c_{m}-c_{d}\right)+\lambda\left(c_{m}+c_{d} r\right)
\end{aligned}
$$

The function $C_{I}(R, M, u)$ can be written in the next form

$$
\begin{aligned}
& C_{I}(M, R, u)= \\
& \sqrt{2 \lambda\left(A(u) \frac{R}{M}+B(u) \frac{M}{R}+C(u) R+D(u) M+E(u)\right)}+ \\
& \lambda u\left(c_{r}-c_{m}-c_{d}\right)+\lambda\left(c_{m}+c_{d} r\right)
\end{aligned}
$$

where

$$
\begin{aligned}
& A(u)=K_{r} h_{m}(1-u)^{2}, B(u)=K_{m}\left(h_{r}+h_{n}\right) u^{2}, \\
& C(u)=K_{r} h_{n}\left(\frac{1}{r}-1\right) u^{2} \\
& D(u)=K_{m} h_{n}\left(\frac{1}{r}-1\right) u^{2} \\
& E(u)=K_{r}\left(h_{r}+h_{n}\right) u^{2}+K_{m} h_{m}(1-u)^{2}
\end{aligned}
$$

and variables $R$ and $M$ are positive integers, and $0 \leq u \leq r$.

The manufacturing and remanufacturing lots are in this case

$$
\begin{gathered}
Q_{m}(M, R, u)=\frac{(1-u) \lambda}{M} . \\
\sqrt{\frac{R K_{r}+M K_{m}}{\frac{h_{r}+h_{n}}{2} u^{2} \lambda \frac{1}{R}+\frac{h_{m}}{2}(1-u)^{2} \lambda \frac{1}{M}+\frac{h_{n}}{2} u^{2} \lambda\left(\frac{1}{r}-1\right)}}
\end{gathered}
$$

and

$$
\begin{aligned}
& Q_{r}(M, R, u)=\frac{u \lambda}{R} . \\
& \sqrt{\frac{R K_{r}+M K_{m}}{\frac{h_{r}+h_{n}}{2} u^{2} \lambda \frac{1}{R}+\frac{h_{m}}{2}(1-u)^{2} \lambda \frac{1}{M}+\frac{h_{n}}{2} u^{2} \lambda\left(\frac{1}{r}-1\right)}} .
\end{aligned}
$$

Function $C_{I}(R, M, u)$ is quasiconvex in $R$ and $M$ and convex in $u$. This property guarantees the existence of optimal solution, as it is prooved by Dobos and Richter [5]. Let us now introduce an auxilliary function $S(R, M, u)$, as follows

$$
\begin{gathered}
S(R, M, u)=A(u) \frac{R}{M}+B(u) \frac{M}{R}+ \\
C(u) R+D(u) M+E(u) .
\end{gathered}
$$

We look for an optimal solution of this function for remanufacturing and manufacturing batches $R$ and $M$. This function is the expression under the square in (1). Due to monotonicity considerations the function $S(R, M, u)$ can be analysed for solving batch sizes $R$ and $M$, where all coefficients $A(u), B(u), C(u)$, $D(u)$ and $E(u)$ are positive. The used meta-model is shown in the appendix of this paper. After some calculation we get the possible set of reuse rate $u$.
Theorem 2 The continuous optimal solution for minimizing the function $C_{I}(R, M, u)$ for $R$ and $M$ is

$$
\begin{aligned}
& \text { (i) } u \leq \min \left\{\frac{\sqrt{K_{r} h_{m}}}{\sqrt{K_{r} h_{m}}+\sqrt{K_{m}\left(h_{r}+h_{n} \frac{1}{r}\right)}} ; r\right\} \\
& R(u)=1, M(u)=\frac{1-u}{u} \sqrt{\frac{K_{r} h_{m}}{K_{m}\left(h_{r}+h_{n}\right)+K_{m} h_{n} \frac{1-r}{r}}}, \\
& C^{R}(u)=u \sqrt{2 \lambda K_{r}\left(h_{r}+\frac{1}{r} h_{n}\right)}+(1-u) \sqrt{2 \lambda K_{m} h_{m}}+ \\
& u \lambda\left(c_{r}-c_{m}-c_{d}\right)+\lambda\left(c_{m}+c_{d} r\right), \\
& \text { (ii) } \frac{\sqrt{K_{r} h_{m}}}{\sqrt{K_{r} h_{m}}+\sqrt{K_{m}\left(h_{r}+h_{n} \frac{1}{r}\right)}} \leq u \leq \\
& \frac{\sqrt{K_{r} h_{m}}}{\sqrt{K_{r} h_{m}}+\sqrt{K_{m}\left(h_{r}+h_{n}\right)-K_{r} h_{n}\left(\frac{1}{r}-1\right)}} \\
& R(u)=1, M(u)=1 \text {, } \\
& C^{T}(u)=\sqrt{2 \lambda\left(K_{r}+K_{m}\right)\left[\left(h_{r}+\frac{1}{r} h_{n}\right) u^{2}+h_{m}(1-u)^{2}\right]}+ \\
& u \lambda\left(c_{r}-c_{m}-c_{d}\right)+\lambda\left(c_{m}+c_{d} r\right), \\
& \text { (iii) } \frac{\sqrt{K_{r} h_{m}}}{\sqrt{K_{r} h_{m}}+\sqrt{K_{m}\left(h_{r}+h_{n}\right)-K_{r} h_{n}\left(\frac{1}{r}-1\right)}} \leq u \leq r \\
& R(u)=u \sqrt{\frac{K_{m}\left(h_{r}+h_{n}\right)}{K_{r} h_{m}(1-u)^{2}+K_{r} h_{n} \frac{1-r}{r} u^{2}}}, \quad M(u)=1 \text {, } \\
& C^{T}(u)=u \sqrt{2 \lambda K_{r}\left(h_{r}+h_{n}\right)}+ \\
& \sqrt{2 \lambda K_{m}\left[(1-u)^{2} h_{m}+u^{2}\left(\frac{1}{r}-1\right) h_{n}\right]}+ \\
& u \lambda\left(c_{r}-c_{m}-c_{d}\right)+\lambda\left(c_{m}+c_{d} r\right) .
\end{aligned}
$$

As we see, the optimal continuous solutions are the same as before. But this formulation of the problem makes it possible to investigate the integer solution of the model (1), as it is made in paper Dobos and Richter [1].

In the next section we outline the integer solution of model (1).

\subsection{Integer solution of model 1}

The optimal continuous solution of problem $(\mathrm{P})$ is on the border of the possible sets of $(R(u), M((u))$, i.e. at least one of the batch is equal to one. The question is whether the optimal integer solution is on the border, or there is optimal integer solution inside of the possible set, i.e. both of the batch numbers are strictly greater than one. To answer the question, we first determine the integer solution on the border of the possible set.

\section{Theorem 3 (Dobos and Richter [1])}

The integer optimal solution for minimizing the function $C_{I}(R, M, u)$ for $R$ and $M$ is

$$
\begin{aligned}
& l u \leq \min \left\{\frac{\sqrt{K_{r} h_{m}}}{\sqrt{K_{r} h_{m}}+\sqrt{K_{m}\left(h_{r}+h_{n} \frac{1}{r}\right)}} ; r\right\} \\
& R^{g}(u)=1, \\
& M^{g}(u)=\left\lfloor\sqrt{\left(\frac{1-u}{u}\right)^{2} \frac{K_{r} h_{m}}{K_{m}\left(h_{r}+h_{n}\right)+K_{m} h_{n} \frac{1-r}{r}}+\frac{1}{4}}\right\rfloor+\frac{1}{2},
\end{aligned}
$$




$$
\begin{aligned}
& 2 \frac{\sqrt{K_{r} h_{m}}}{\sqrt{K_{r} h_{m}}+\sqrt{K_{m}\left(h_{r}+h_{n} \frac{1}{r}\right)} \leq u \leq} \\
& \frac{\sqrt{K_{r} h_{m}}}{\sqrt{K_{r} h_{m}}+\sqrt{K_{m}\left(h_{r}+h_{n}\right)-K_{r} h_{n}\left(\frac{1}{r}-1\right)}} \leq u \leq r \\
& R^{g}(u)=1, M^{g}(u)=1, \\
& \left.3 \frac{\sqrt{K_{r} h_{m}}}{\sqrt{K_{r} h_{m}}+\sqrt{K_{m}\left(h_{r}+h_{n}\right)-K_{r} h_{n}\left(\frac{1}{r}-1\right)}} \leq u+\frac{1}{4}\right\rfloor+\frac{1}{2}, \\
& R^{g}(u)=\left\lfloor\sqrt{u^{2} \frac{K_{m}\left(h_{r}+h_{n}\right)}{K_{r} h_{m}(1-u)^{2}+K_{r} h_{n} \frac{1-r}{r} u^{2}}+\frac{1}{4}}\right. \\
& M^{g}(u)=1,
\end{aligned}
$$

Notation $|x|$ denotes the greatest integer not higher than value $x$. We will not prove this theorem, the proof is can be seen in paper of Dobos and Richter [5]. The border batch numbers $\left(R^{g}(u)\right.$, $\left.M^{g}(u)\right)$ are optimal for some cases, but there exist optimal solutions inside of the possible set of batch numbers. In the next section we show some example to demonstrate the reults of the theorem.

It can be proven that the inventory related cost difference between the a border solution $\left(R^{g}(u), M^{g}(u)\right)$ and an optimal solution inside of the possible set is not higher than that of 2.1 percent (Dobos and Richter [1]). The optimal integer solution can be calculated, as

$$
\begin{gathered}
Q_{m}\left(M^{g}(u), R^{g}(u), u\right)=\frac{(1-u) \lambda}{M} \\
\sqrt{\frac{R^{g}(u) K_{r}+M^{g}(u) K_{m}}{\frac{h_{r}+h_{n}}{2} u^{2} \lambda \frac{1}{R^{g}(u)}+\frac{h_{m}}{2}(1-u)^{2} \lambda \frac{1}{M^{g}(u)}+\frac{h_{n}}{2} u^{2} \lambda\left(\frac{1}{r}-1\right)}},
\end{gathered}
$$

and

$$
\frac{Q_{r}\left(M^{g}(u), R^{g}(u), u\right)=\frac{u \lambda}{R}}{\sqrt{\frac{R^{g}(u) K_{r}+M^{g}(u) K_{m}}{\frac{h_{r}+h_{n}}{2} u^{2} \lambda \frac{1}{R^{g}(u)}+\frac{h_{m}}{2}(1-u)^{2} \lambda \frac{1}{M^{g}(u)}+\frac{h_{n}}{2} u^{2} \lambda\left(\frac{1}{r}-1\right)}} .}
$$

\subsection{Numerical examples}

\section{Example 1.}

Let the parameters be in the next example:

$\lambda=1.000$ piece/year, $r=0.9, u=0.5$,

$K_{m}=\$ 750, K_{r}=\$ 100$,

$h_{m}=\$ 200, h_{r}=\$ 50$,

$h_{n}=\$ 20$,

$c_{m}=\$ 20, c_{r}=\$ 15, c_{d}=\$-35$.

In this example the remanufacturing firm sells the disposed items. The optimal continuous solution for this problem is:

$$
R^{o}=1.611, M^{o}=1,
$$

$Q_{r}^{o}=184.113$,

$Q_{m}^{o}=114.285, T^{o}=0.368$ year.

The inventory holding costs are 10,579.1. The linear costs are

$-8,500$. The total costs are 2,079.1.

The optimal integer solution is:

$R^{o}=2, M^{o}=1, Q_{r}^{o}=204.896$,

$Q_{m}^{o}=102.448, T^{o}=0.41$ year.
The inventory holding costs are 10,615.1. The linear costs are $-8,500$. The total costs are 2,115.1.

The inventory related costs of the integer solution are 0.3 percent higher than that of costs of continuous solution.

Example 2.

Let the parameters be in the following example:

$\lambda=1.000$ piece/year, $r=0.9, u=0.48$,

$K_{m}=\$ 750, K_{r}=\$ 100$,

$h_{m}=\$ 200, h_{r}=\$ 50, h_{n}=\$ 20$,

$c_{m}=\$ 20, c_{r}=\$ 15, c_{d}=\$-35$.

The difference of these two examples are that the reuse rate is lower in the following problem. In this example the remanufacturing firm sells the disposed items. The optimal continuous solution for this problem is:

$$
\begin{aligned}
& R^{o}=1.489, M^{o}=1, Q_{r}^{o}=176.573, \\
& Q_{m}^{o}=128.467, T^{o}=0.368 \text { year. }
\end{aligned}
$$

The inventory holding costs are 10,845.2. The linear costs are $-10,700$. The total costs are 145.2.

The optimal integer solution is:

$R^{o}=3, M^{o}=2, Q_{r}^{o}=174.747$,

$Q_{m}^{o}=126.206, T^{o}=0.728$ year.

The inventory holding costs are 10,887.6. The linear costs are $-10,700$. The total costs are 187.6.

The inventory related costs of the integer solution are 0.4 percent higher than that of costs of continuous solution.

The solution of the border line is

$$
R^{o}=2, M^{o}=1, Q_{r}^{o}=204.441, Q_{m}^{o}=110.739, T^{o}=0.426
$$
year.

The inventory holding costs are 10,910.8. The linear costs are $-10,700$. The total costs are 210.8. The optimal integer solution for the total costs are 12.4 percent higher than that of of the integer solution on the border line. The isocost line of the optimal solution is shown on Fig. 4 .

\section{Calculation of the optimal reuse rate $u$}

We determine the optimal reuse rate from the continuous cost function:

$$
\begin{gathered}
C^{R}(u)= \\
u \sqrt{2 \lambda K_{r}\left(h_{r}+\frac{1}{r} h_{n}\right)}+(1-u) \sqrt{2 \lambda K_{m} h_{m}}+ \\
u \lambda\left(c_{r}-c_{m}-c_{d}\right)+\lambda\left(c_{m}+c_{d} r\right), \\
0 \leq u<\leq u_{1}(r) \\
\sqrt{2 \lambda\left(K_{r}+K_{m}\right)\left[\left(h_{r}+\frac{1}{r} h_{n}\right) u^{2}+h_{m}(1-u)^{2}\right]}+ \\
u \lambda\left(c_{r}-c_{m}-c_{d}\right)+\lambda\left(c_{m}+c_{d} r\right), \\
u_{1}(r) \leq u \leq u_{2}(r) \\
u \sqrt{2 \lambda K_{r}\left(h_{r}+h_{n}\right)}+\sqrt{2 \lambda K_{m}\left[(1-u)^{2} h_{m}+u^{2}\left(\frac{1}{r}-1\right) h_{n}\right]}+ \\
u \lambda\left(c_{r}-c_{m}-c_{d}\right)+\lambda\left(c_{m}+c_{d} r\right), \\
u_{1}(r) \leq u \leq r
\end{gathered}
$$

In this model we have assumed, that $r_{2} \leq r$. (If return rate $r$ not greater than $r_{2}$, then we have one or two interval to look for the optimal reuse rate.) It means that we have three types batch numbers. We have seen that function $C^{R}(u)$ is continuous and continuously differentiable on interval $0 \leq u \leq r$. It is obvious 


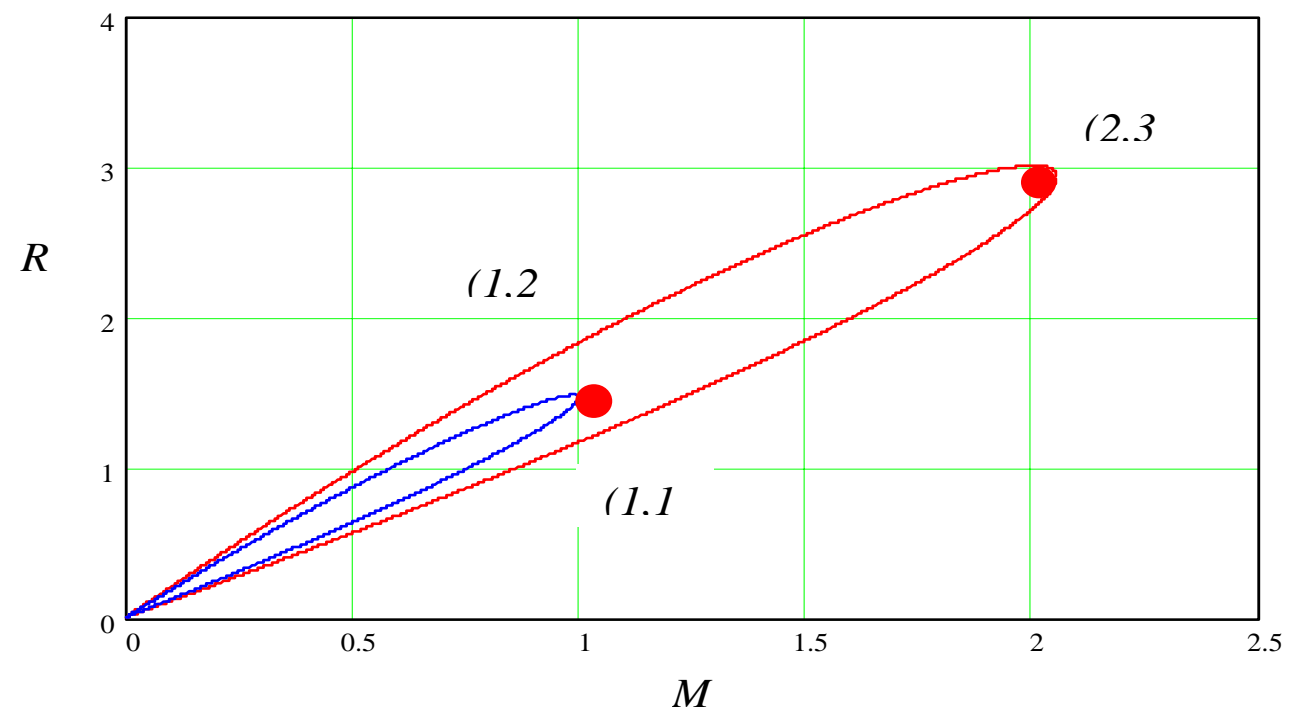

Fig. 4. The optimal integer solution inside of the possible set

that the cost function is linear on the first interval and convex for all possible reuse rate.

Let us assume that the setup cost of manufacturing is higher than that of remanufacturing, i.e. $K_{m}>K_{r}$. A second assumption is that $h_{m}>h_{r}+h_{n}$, i.e. the holding costs of newly manufactured item are higher than that of sum of holding costs of remanufactured and non-serviceable items. This assumption makes the remanufacturing process cost efficient. And last $c_{m}+c_{d}>c_{r}$, i.e. the sum of manufacturing and disposal costs are higher than that of remanufacturing costs, if the disposal cost is positive. The disposal cost coefficient can be negative, as well. If this coefficient is negative, then it can be sold on a second market of used products. With these assumption we can characterize the optimal reuse rate.

Lemma 4 If the disposal costs of returned, non-serviceable items are positive, i.e. $c_{d}>0$, then the optimal reuse rate is equal to the return rate: $u^{o}=r$.

The proof of the lemma is very simple. The function $C^{R}(u)$ is contiuous and convex, so it is enough to examine the function in point $r$. If this function is decreasing in ponit $r$, then the cost function $C^{R}(u)$ is decreasing for all possible reuse rate $u$. The next inequality holds for all possible reuse rate $u$ :

$$
\begin{gathered}
u \sqrt{2 \lambda K_{r}\left(h_{r}+h_{n}\right)}+ \\
u \lambda<\sqrt{2 \lambda K_{m}\left[(1-u)^{2} h_{m}+u^{2}\left(\frac{1}{r}-1\right) h_{n}\right]}+ \\
u \lambda\left(c_{m}+c_{d}\right) .
\end{gathered}
$$

With this property we have finished our investigation of the problem.

\section{Conclusion}

In this paper we have resolved a product recovery problem with average inventory related costs. We have shown to methods to determine the optimal decision variables. We have presented a meta-model to calculate the optimal integer manufacturing and remanufacturing batch numbers. It was shown that the optimal number of both manufacturing and remanufacturing sizes can be strictly greater than one, for some cost papameter structure. The solution of the model considers exclusively such cases when both batch numbers are greater than zero, but not integer.

The managerial implication of the model is that the number of manufacturing and remanufacturing batches depend on the collection and reuse rate. If the collection rate is high, then it is economical to satisfy the demand from remanufacturing and the required items to manufacture. And in other case, if the collection rate is low, then the important activity is the manufacturing. An other implication of the model is that all returned, used products are free resources of the firm, so it is recommended to reuse all collected and returned items. The model can be used in the managerial praxis in case of relatively constant demand and return rates. In this case the lot sizes for manufacturing and remanufacturing are a good estimation of the managerial batches.

\section{References}

1 Dobos I, Richter K, The integer EOQ repair and waste disposal model-further analysis, Central European Journal of Operations Research, 8, (2000), 173-194.

2 Nahmias $\mathbf{N}$, Rivera $\mathbf{H}$, A deterministic model for repairable item inventory system with a finite repair rate, International Journal of Production Research, 17, (1979), 215-221, DOI 10.1080/00207547908919609.

3 Richter K, The EOQ repair and waste disposal model with variable setup numbers, European Journal of Operational Research, 96, (1996), 313-324, DOI 10.1016/0377-2217(95)00276-6.

4 Schrady D A, A deterministic inventory model for repairable items, Naval Research Logistic Quarterly, 14, (1967), 391-398, DOI 10.1002/nav.3800140310.

5 Teunter R H, Economic Ordering Quantities for Recoverable Item Inventory Systems, Naval Research Logistics, 48, (2001), 484-495, DOI 10.1002/nav.1030. 


\section{Appendix}

The following auxiliary function $S(m, n)$ can be derived from the cost function $C_{I}(M, R, u)$

$$
S(m, n)=A \frac{m}{n}+B \frac{n}{m}+C m+D n+E .
$$

This function convex in $m$ and in $n$, strictly quasiconvex in $(m, n)$, because of the positivity of the coefficients.

The fractional program of minimizing the function (1) for arbitrary real inputs,

$S(m, n) \rightarrow \min$,

$(m, n) \in S_{G}=\{(m, n): m, n \in\{1,2, \ldots\}\}$,

i. e. the problem of finding an optimal $(m, n)$ is discussed below. The program will be shortly called "integer problem".

The relaxed fractional program

$S(m, n) \rightarrow \min$,

$(m, n) \in S=\{(m, n):(m, n) \geq 1\}$

has been studied in paper [1]. It will be called the "continuous problem". First some properties will be presented here.

Theorem 5 There are three cases of optimal continuous solutions $(m, n)$ and minimum cost expressions $S^{*}$ for the function (5) on $S$ :

$$
\begin{array}{ll}
1 & B \geq A+C \\
& \left(m^{*}, n^{*}\right)=\left(\sqrt{\frac{b}{a+c}}, 1\right), \\
& S^{*}=2 \sqrt{b(a+c)}+d+e, \\
2 & A-D \leq B \leq A+C \\
& \left(m^{*} n^{*}\right)=(1,1) \\
& S^{*}=A+B+C+D+E \\
3 & B \leq A-C\left(m^{*}, n^{*}\right)=\left(1, \sqrt{\frac{a}{b+d}}\right), \\
& S^{*}=2 \sqrt{a(b+d)}+c+e .
\end{array}
$$

